\title{
Balloon catheter dilatation of coarctation of the aorta in young infants
}

\author{
JOHN P FINLEY, ROLAND G BEAULIEU, MAURICE A NANTON, DOUGLAS L ROY \\ From the Division of Pediatric Cardiology, Izaak Walton Killam Hospital for Children and Dalhousie University, \\ Halifax, Nova Scotia, Canada
}

SUMMARY In four young infants with symptomatic coarctation of the aorta the narrow aortic segment was dilated with a balloon angioplasty catheter. Three of the infants also had appreciable heart defects and one infant had undergone surgery before and had had one previous dilatation. Dilatation was well tolerated and good femoral pulses appeared in all cases, but these disappeared over one to seven days. Despite this, two patients showed some symptomatic improvement over four months. One patient died after perforation of the aorta by an angiographic catheter after dilatation.

Relieving coarctation in young infants by catheter dilatation appears incomplete, but it may give symptomatic improvement. Further trial of the procedure will determine whether patients who have undergone surgery and those who have not may benefit. Careful attention to technique and avoidance of manipulations after dilatation are essential if complications are to be avoided.

The treatment of symptomatic coarctation in newborn infants remains controversial. Severe heart failure or hypertension, often in the presence of other cardiac lesions, frequently fail to respond to medical treatment alone. Unfortunately, surgery in young infants has been associated with appreciable mortality especially when additional defects are present. Recurrence of coarctation has been reported in $10-30 \%$ of survivors despite recent improvements in surgical technique..$^{1-4}$

The high incidence of recoarctation and the frequent need for repeat surgery with its associated risks have made alternatives to early surgical treatment highly desirable. Recent reports of balloon dilatation of coarctation in infancy ${ }^{5-7}$ have suggested at least temporary success in a few young infants, and, although experience is limited, further evaluation is clearly indicated. We describe our experience with four infants, one of whom underwent the procedure twice, with particular emphasis on the complications encountered.

\section{Patients and methods}

All infants underwent diagnostic cardiac catheterisa- tion, including retrograde aortogram, either as a separate procedure or at the same time as the dilatation. On four occasions a percutaneous technique was used for dilatation; on one occasion the right femoral artery was exposed directly. A Meditech (Watertown, MA 02172 ) balloon angioplasty catheter (4 French, balloon length $1.5 \mathrm{~cm}$, balloon diameter $4-6 \mathrm{~mm}$ ) which was twice the diameter of the coarctation, as in the method of Lock et al. ${ }^{8}$ was advanced over a $0.45 \mathrm{~mm}$ guidewire to the approximate position of the coarctation. The positioning of the balloon was confirmed with a test inflation to $1 \mathrm{~atm}$, with the narrow segment producing an hourglass configuration of the balloon. Diatrizoate meglumine diluted with normal saline was used to inflate the balloon. Dilatation was performed by inflating the balloon to $6 \mathrm{~atm}$ for $30 \mathrm{~s}$, with continuous arch pressure and fluoroscopic monitoring. The balloon was then rapidly deflated and the catheter withdrawn, leaving the guidewire tip above the coarctation. In three cases an angiographic catheter was then introduced over the wire for a repeat aortogram, although this procedure has now been abandoned (see discussion). In all cases heparin 100 units $/ \mathrm{kg}$ was given intravenously immediately after placement of the arterial catheter. After the procedure patients were observed in the intensive care unit for 24 hours. 

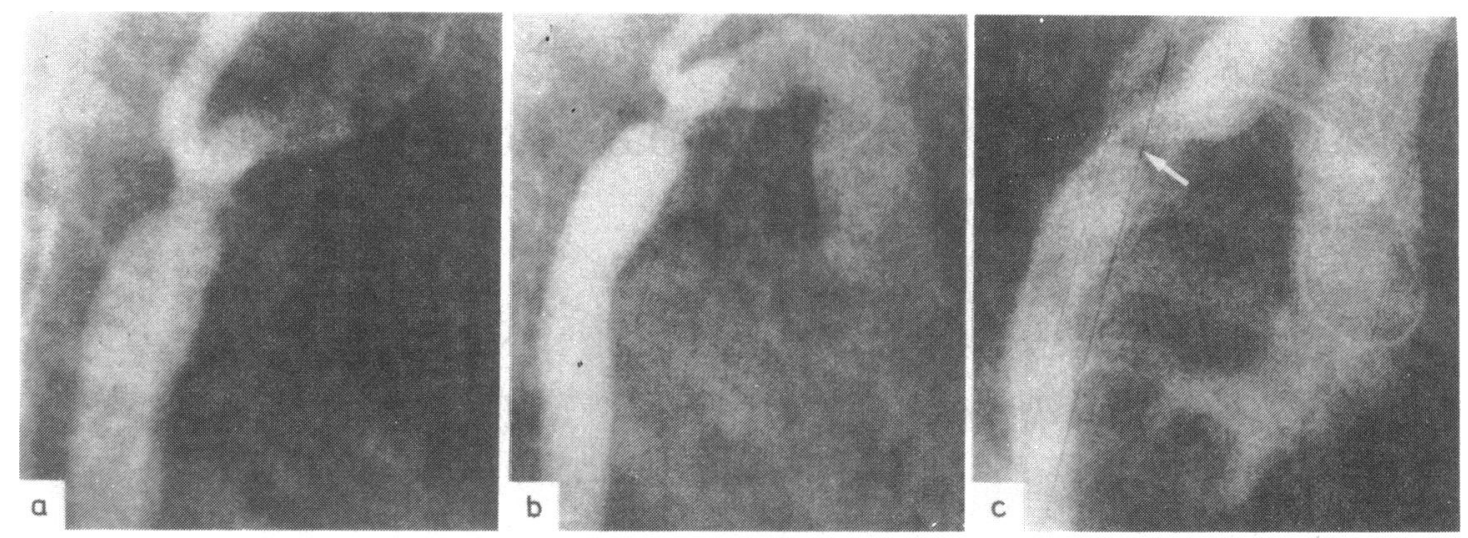

Fig. 1 Case 1. Lateral aortograms. (a) Immediately after first dilatation aged 3 months. (b) Immediately before second dilatation, aged 4 months, showing increased narrowing compared with (a). (c) Slightly oblique projection four months after second dilatation, showing persistent stenosis but less pronounced than in (b) (measured gradient $15 \mathrm{~mm}$ ).

\section{Results}

\section{CASE 1}

Catheterisation in a 10 day old baby boy with considerable congestive failure and absent femoral pulses showed severe coarctation of the aorta with a large ventricular septal defect, aortic stenosis, and a patent ductus arteriosus. A patch aortoplasty was performed immediately along with ligation of the ductus arteriosus. Although femoral pulses were initially good, the infant failed to gain weight, his femoral pulses gradually disappeared, and congestive failure grew worse despite treatment. At three months of age cardiac catheterisation showed recurrence of the coarctation and a small shunt. Dilatation of the stenotic area was performed percutaneously via the right femoral artery with a $4 \mathrm{~mm}$ diameter balloon. There were no complications. Angiography after dilatation showed little change in the coarctation (Fig. 1), and the femoral pulses, which were good immediately after the procedure, deteriorated over 24 hours. Congestive failure and poor feeding continued and at $5 \frac{1}{2}$ months of age dilatation was repeated with a $6 \mathrm{~mm}$ diameter balloon. Ascending aortic pressures were as follows: before dilatation $112 / 70 \mathrm{~mm} \mathrm{Hg}$ with a peak systolic gradient of $26 \mathrm{~mm} \mathrm{Hg}$ across the coarctation; after dilatation $90 / 58 \mathrm{~mm} \mathrm{Hg}$, gradient $12 \mathrm{~mm} \mathrm{Hg}$. Angiography indicated a slight dilatation of the coarctation. No complications were encountered. Strong femoral pulses were present after the procedure but weakened over one week. Congestive failure and feeding, however, improved considerably. Repeat catheterisation four months after dilatation showed a gradient of $15 \mathrm{~mm} \mathrm{Hg}$ across the coarctation, which, on angiography, appeared improved (Fig. 1).

\section{CASE 2}

A full term baby boy presented with a murmur, absent femoral pulses, and congestive heart failure at 10 days of age. Systolic blood pressure in the right arm was $170 \mathrm{~mm} \mathrm{Hg}$. Cardiac catheterisation confirmed a narrow coarctation of the aorta (Fig. 2). Because of persistent hypertension, at 4 weeks of age dilatation of the coarctation was performed percutaneously from the right femoral artery with a $4 \mathrm{~mm}$ diameter balloon. Systolic pressure proximal to the coarctation decreased from $150 \mathrm{~mm} \mathrm{Hg}$ before dilatation to $120 \mathrm{~mm} \mathrm{Hg}$ afterwards. This was associated with the appearance of a good femoral pulse. An aortogram showed some dilatation of the stenotic area. (Fig. 2). The procedure was well tolerated, but over four days the femoral pulse gradually disappeared. Because the right brachial blood pressure remained below $130 \mathrm{~mm} \mathrm{Hg}$ and the baby was growing well, he was discharged two weeks later. Over the next two months he continued to thrive, despite mild congestive failure, absent femoral pulses, and a right brachial blood pressure of $120 \mathrm{~mm} \mathrm{Hg}$. Surgical correction is planned at 6-8 months of age.

\section{CASE 3}

A full term baby boy developed severe congestive failure and cyanosis in the first week. Soon after referral he had a cardiac arrest, from which he was quickly resuscitated with the aid of intravenous prostaglandin E1. Emergency cardiac catheterisation showed a double outlet right ventricle with subpulmonary ventricular septal defect and coarctation of the aorta. A balloon atrial septostomy was performed, and because of severe congestive failure and poor cardiac output dilatation of the coarctation was carried out with puncture 


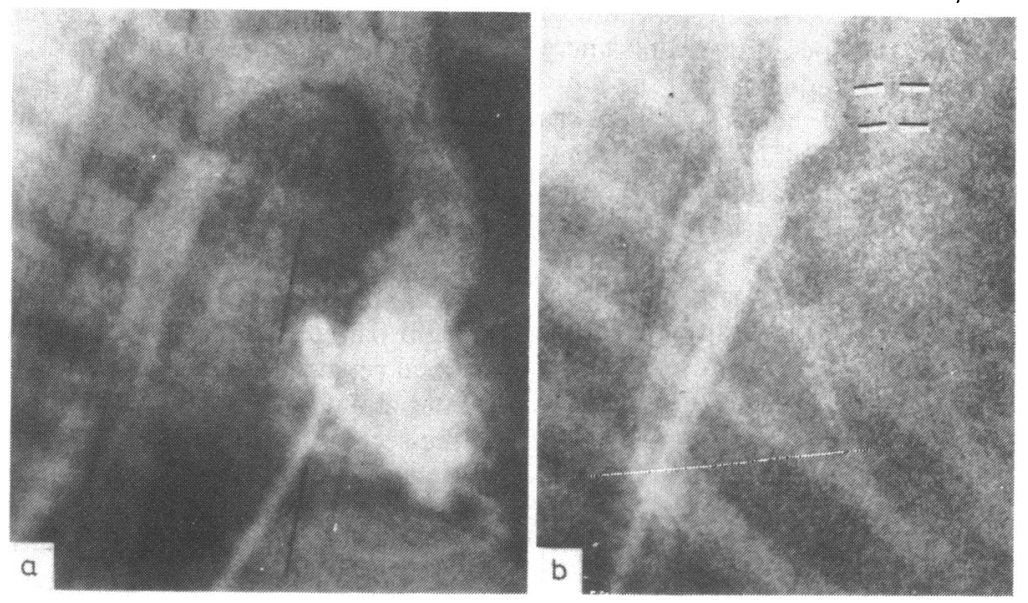

Fig. 2 Case 2. Lateral aortograms. (a) Before dilatation (aged 3 weeks), (b) immediately after dilatation (aged 4 weeks), showing decreased stenosis. Position of aortic arch shown by dashed lines.

of the exposed femoral artery. A $5 \mathrm{~mm}$ diameter balloon was used, and a good left femoral pulse appeared with dilatation, which was well tolerated. The pulses gradually disappeared over $\mathbf{4 8}$ hours, and since severe congestive failure continued coarctectomy and end to end anastomosis were performed at four weeks. Subsequently, femoral pulses have remained excellent, although pulmonary arterial banding became necessary at 6 weeks of age. Histalogical examination of the excised segment of the aortia showed focal disruption of the internal elastic fibres and fibrous replacement of a sector of media (Fig. 3).

\section{CASE 4}

At 1 week of age a baby boy presented with mild congestive heart failure, absent femoral pulses, and poor feeding. Cardiac catheterisation showed coarctation of the aorta with ventricular septal defect. Congestive failure was controlled with digoxin and diuretics, feeding did not improve, and the right brachial blood pressure gradually increased to $150 \mathrm{~mm} \mathrm{Hg}$. At five weeks of age dilatation of the coarctation was performed. An initial aortic arch angiogram was produced, and dilatation of the coarctation was uneventful, being accompanied by a fall in systolic pressure from 170 to $120 \mathrm{~mm} \mathrm{Hg}$. While removing the catheter, despite efforts to retain the guidewire in the aortic arch, the wire was withdrawn into the abdominal aorta. An attempt was made to manipulate a 4 French Cook angiographic catheter into the position of the coarctation without using the wire. Aortic pressure decreased, however, and a rapid injection of contrast medium showed extravasation of dye into the pericardial sac. Cardiac output gradually decreased, and

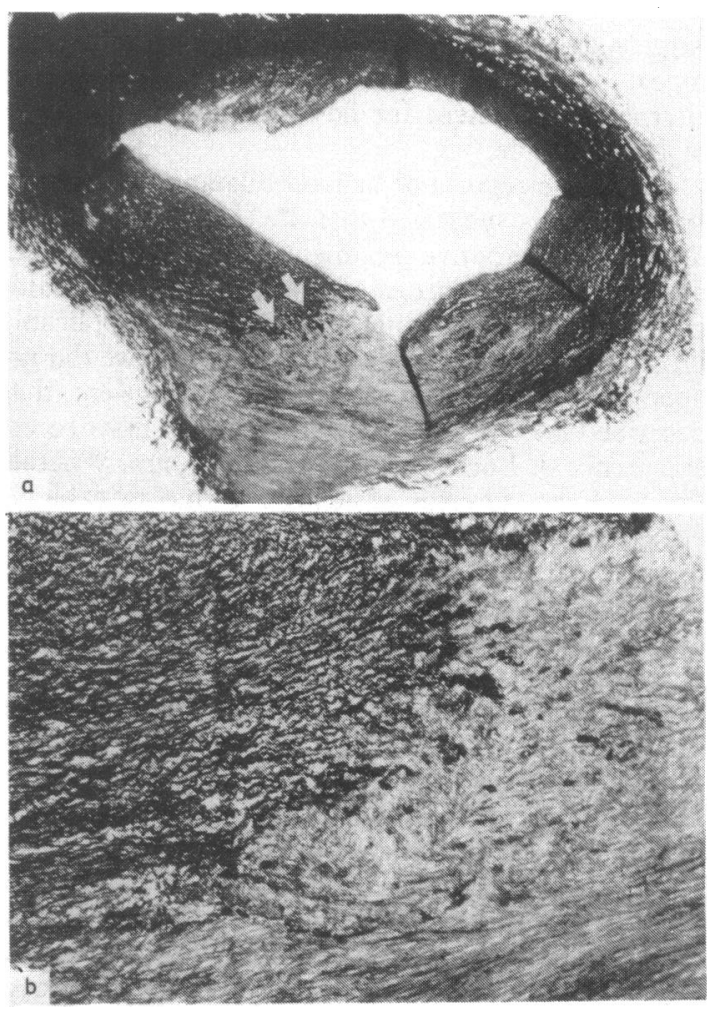

Fig. 3 Case 3. (a) Elastic stain of section of aorta at site of coarctation three weeks after dilatation, showing disruption of elastic lamina and focal fibrous replacement affecting both intima and media (arrows) ( $\times$ 37) (b) High power of border between fragmented elastic lamina and fibrous tissue (elastic stain, $\times 150$ ). 
resuscitation was rapidly started. Within 20 minutes the patient was moved to the operating room, where the site of bleeding was quickly identified at the junction of the ductus arteriosus and the aorta. There was no evidence of blood in the thorax. Pericardial tamponade was relieved, but despite prolonged direct cardiac massage mechanical and electrical activity did not return.

At necropsy the perforation site was confirmed at the origin of the ductus. There was clear evidence on microscopy of intimal tearing at the site of coarctation, and histological sections showed the expected rupture of elastic fibres. Bleeding appeared to have tracked through a pericardial reflection along the ductus arteriosus and into the pericardial space.

\section{Discussion}

Both medical and surgical treatment of symptomatic coarctation in newborn infants have considerable disadvantages. Medical treatment accepts the presence of hypertension for months, with the risk of pathological effects on the developing aorta. On the other hand, surgical results are uncertain in young infants, and repeat operation is often necessary. ${ }^{1-4}$ Clearly, an alternative treatment for newborn infants would be highly desirable.

Early case reports of balloon dilatation of coarctation in infants suggested that the technique might be used as an alternative treatment. ${ }^{56}$ Although limited, our experience indicates several problems associated with the technique, which may restrict its applicability. In the two surviving infants on whom we did not operate relief of stenosis was more transient than described in early reports, ${ }^{56}$ although the more recent experience of Lock et al. ${ }^{7}$ is similar to ours. Whether the tendency towards restenosis is due to a highly elastic nature of the coarctation segment in young infants, scarring, residual capability for contraction in the juxta ductal region, or some other factor is not clear. More extensive dilatation may not be a realistic alternative since the balloon diameter used in the present study is probably the largest compatible with safety during dilatation and during introduction through the very small femoral artery of the infant. Despite the rapid disappearance of femoral pulses after dilatation in infants with unoperated coarctation, symptomatic improvement may still occur, as in case 1 , allowing a delay of operation until the child has reached a size where results are more favourable. In cases of complex coarctation even transient improvement produced by dilatation may allow stabilisation of the patient pending more definitive treatment.

The outlook for patients who have undergone surgery may be better. Early enthusiasm for the technique was based partly on favourable experience with surgically created arterial stenosis in lambs, ${ }^{8}$ in which the stenotic site would have had considerable scarring. It may be that a scarred coarctation site is the most favourable for successful dilatation. In our patient who had undergone surgery we achieved a satisfactory result with dilatation after a balloon of appropriate size had been used (the initial attempt used a size smaller than that described in the protocol). This agrees with the experience of Lock et al. ${ }^{7}$ in eight patients. Although some patients will require surgical repair later, the delay provided by dilatation should allow for growth and a more successful result at the second operation.

Review of histological sections of the dilated coarctation in two patients showed both early and late effects of dilatation. In case 4 the immediate effects were several areas of focal disruption of endothelium and elastic lamina, with haemorrhagic infiltrates similar to those seen after dilatation in lambs. ${ }^{8}$ In case 3 (Fig. 3) sections obtained three weeks after dilatation showed disruption of the elastic lamina localised to a small sector, but damage extended through both intima and media, as indicated by the area of fibrous replacement. That intimal and medial damage is necessary for successful dilatation was recognised by Lock et al ${ }^{8}$ Chronic weakening of the aortic wall was not apparent experimentally, although the wall can easily be perforated 'by a catheter, as in case 4 . Further histological studies should show whether localised or widespread fibrous replacement is more common and provide clues to the mechanism of recoarctation after dilatation.

Lock et al. ${ }^{8}$ paid much attention to the safety of the dilatation procedure, but this has unfortunately received less emphasis in the two patient case reports. ${ }^{67}$ While Lock et al. ${ }^{8}$ made deliberate attempts to fracture the aorta of lambs, perforation occurred only with dilating balloons with diameters more than three times the diameter of the coarctation. The safety of the smaller balloon has been confirmed in patients. More important, however, is the danger of perforation of the dilated segment after the procedure by a catheter or wire, as occurred in case 4 . While a catheter can be safely repositioned above the site of dilatation if passed over a wire left in place after dilatation, ${ }^{8}$ we now believe that no catheter should be passed through the dilated site after the procedure. For confirmation of a successful dilatation, gradients can be measured through the lumen of the dilating catheter as it is withdrawn, and a repeat angiogram of the coarctation can be produced from a venous catheter.

We are also concerned that very young patients may have an additional risk relating to the aortic origin of the ductus arteriosus. Ductus tissue in these patients is extremely friable, and being next to the coarctation 
it may provide a site for rupture of the aortic wall during initial wire placement before dilatation or during the procedure itself.

In conclusion, we believe that balloon dilatation of coarctation deserves further evaluation in infants. but with certain precautions during and after the procedure. An immediate decrease in gradient and appearance of normal femoral pulses do not ensure a successful result. Further follow up will determine whether patients who have been operated on and those who have not been operated on will benefit from dilatation.

We thank Dr V Krause for supplying the material for histological examination and Dr J Lock for reviewing the manuscript.

\section{References}

1 Hesslein PS, Gutgesell HP, McNamara DG. Prognosis of symptomatic coarctation of the aorta in infancy. Am $\mathcal{Y}$ Cardiol 1983; 51: 299-303.

2 Fyler DC, Buckley LP, Hellenbrand WE, et al. Report of the New England Regional Infant Cardiac Program. Pediatrics 1980; 65 (suppl): 375-461.
3 Waldhausen JA, Whitman V, Werner JC, Pierce WS. Surgical intervention in infants with coarctation of the aorta. F Thorac Cardiovasc Surg 1981; 81: 323-5.

4 Kamau P, Miles V, Toews W, et al. Surgical repair of coarctation of the aorta in infants less than six months of age. F Thorac Cardiovasc Surg 1981; 81: 171-9.

5 Singer MI, Rowen M, Dorsey TJ. Transluminal aortic balloon angioplasty for coarctation of the aorta in the newborn. Am Heart f 1982; 103: 131-2.

6 Sperling DR, Dorsey TJ, Rowen M, Gazzaniga AB. Percutaneous transluminal angioplasty of congenital coarctation of the aorta. Am $\mathcal{F}$ Cardiol 1983; 51: 562-4.

7 Lock JE, Bass JL, Amplatz K, Fuhrman BP, Castaneda-Zuniga W. Balloon dilation angioplasty of aortic coarctations in infants and children. Circulation 1983; 68: $109-16$.

8 Lock JE, Niemi T, Einzig S, Amplatz K, Burke B, Bass JL. Transvenous angioplasty of experimental branch pulmonary artery stenosis in newborn lambs. Circulation 1981; 64: 886-93.

Requests for reprints to Dr J P Finley, 5850 University Avenue, Halifax, Nova Scotia, Canada B3J 3 G9. 